\title{
Risk factors for mortality among patients admitted with upper gastrointestinal bleeding at a tertiary hospital: a prospective cohort study
}

\author{
Sibtain M. Moledina* (i) and Ewaldo Komba
}

\begin{abstract}
Background: Upper gastrointestinal bleeding (UGIB) is a common gastrointestinal emergency, which is potentially fatal. Proper management of UGIB requires risk-stratification of patients which can guide the type and aggressiveness of management. The aim of this was study was identify the causes of UGIB and factors that increase the risk of mortality in these patients.

Methods: This was a prospective cohort study conducted over a period of seven months at a tertiary hospital. Adults admitted with UGIB were included in the study. Demographic data, laboratory parameters and endoscopic findings were recorded. Patients were then followed up for 60 days to identify the occurrence of mortality. Chi-square tests and cox-regression was used to determine association between risk factors and mortality in the bivariate and multivariate analysis, respectively.

Results: A total of 170 patients with UGIB were included. Males accounted for the majority (71.2\%). Median age of the study population was 40.0 years. Chronic liver disease was present in $30.6 \%$ of study patients. The most common cause of UGIB among the 86 patients who underwent endoscopy was oesophageal varices (57\%), followed by peptic ulcer disease (18\%) and gastritis (10\%). Mortality occurred in 57 patients (33.5\%) and was significantly higher in patients with high white blood cell count (HR 2.45, p 0.011), raised serum alanine aminotransferase (HR 4.22, p 0.016), raised serum total bilirubin (HR 5.79, p 0.008) and lack of an endoscopic procedure done (HR 4.40, $p<0.001$ ). Rebleeding was reported in 12 patients (7.1\%) and readmission due to UGIB in 4 patients (2.4\%)

Conclusions: Oesophageal varices was the most common cause of UGIB. One-third of patients admitted with upper gastrointestinal bleeding died within 60 days of admission, signifying a high burden. Rebleeding and readmission rates were low. A high WBC count, raised serum ALT, raised serum total bilirubin and a lack of endoscopy were independent predictors of mortality. These findings can be used to risk-stratify patients who may benefit from early and more aggressive management.
\end{abstract}

Keyword: Upper gastrointestinal bleeding mortality Tanzania

\footnotetext{
* Correspondence: sibumole@hotmail.com

Department of Internal Medicine, Muhimbili University of Health and Allied

Sciences, P.O. Box 65001, Dar es Salaam, Tanzania
} 


\section{Background}

Upper Gastrointestinal Bleeding is defined as hemorrhage originating from anywhere between the esophagus and the ligament of Treitz [1]. It is one of the most common gastrointestinal emergencies, with an average mortality rate of $10 \%[2,3]$. Despite advances in the diagnosis and management of UGIB, the mortality rate has not changed significantly in the last 50 years [2-4].

Effective management requires use of a riskstratification tool to categorise patients into low-risk and high-risk group, which can be used to guide treatment and follow up [3].

Many risk factors for mortality have been found from different studies. A number of risk scores have been developed also. The most frequently used risk scoring system is the Rockall score which was developed in 1996. This score assesses the risk of death following UGIB and incorporates the patient's age, systolic blood pressure, heart rate, presence of other comorbidities and endoscopic findings [3]. Another frequently used scoring system is the Glasgow Blatchford Score [3].

Upper GI bleeding continues to be a significant cause of morbidity and mortality in Tanzania. For example, a one restrospective study enrolled 130 admitted patients with UGIB within two years [5]. Similarly, in another study in northern Tanzania, of all patients who underwent a fiberoptic upper GI endoscopy, $18.7 \%$ were found to have evidence of UGIB [6]. Oesophageal varices and peptic ulcer disease (PUD) being the most common causes in both these studies. Studies in Tanzania have shown a mortality rate ranging between 10 and $17 \%$ of patients admitted with UGIB [5-7].

This study was done at Muhimbili National Hospital $(\mathrm{MNH})$ in Tanzania, which is the largest tertiary referral hospital currently in the country. No such studies had previously been done from this setting. Only one study was found that was prospectively done to find the risk factors of mortality among patients with UGIB. This study was aimed at identifying the causes of UGIB and risk factors for mortality in these patients, which would help better risk-stratify patients and guide management more efficiently, particularly in resource limited areas.

\section{Methods}

This was a prospective, cohort study where participants were recruited consecutively on admission from June 2015 to January 2016 and followed up for 60 days from admission.

The study population were all patients admitted to MNH due to UGIB. MNH is one of the four tertiary hospitals in the country. It also serves as a tertiary level referral for three municipal hospitals in Dar es Salaam. MNH admits patients with upper gastrointestinal bleeding primarily at Mwaisela Ward, which serves as the Internal
Medicine ward for the hospital. The hospital receives approximately 25 to 30 patients a month with acute upper gastrointestinal bleeding. The patients are initially seen at the Emergency Medicine Department of the hospital and are then shifted to the medical ward after stabilization, where they are then managed accordingly. Facility for upper gastrointestinal endoscopy is available during official work hours and is performed by trained endoscopists in the department of Gastroenterology.

A target sample size of 100 patients was established based on sample size calculation with a confidence interval of $95 \%$, a $5 \%$ margin of error and expected incidence of $10 \%$ [7].

\section{Data collection process}

All patients were entered into the study after an informed consent. Relevant data on demographics, medical history and comorbidities was collected on admission by interviewing the study participants or their guardians if the study participants were not able to answer. Admission vital signs from the Emergency Medicine Department (blood pressure, pulse rate, respiratory rate and oxygen saturation) were also recorded. Data on comorbidities was either confirmed by the patients themselves or through their records. Blood samples for laboratory tests were collected within $24 \mathrm{~h}$ of admission. Upper GI endoscopy was also done by trained personnel in the Gastroenterology Unit for those who could afford to pay for it in order to identify the cause and severity of the bleeding. (Approximately \$60).

All patients were then followed up while admitted in the ward, thereafter two-weekly follow-up was done after discharge up to 60 days post-initial admission or death.

UGIB was defined as any acute episode of vomiting blood or passing melena stool in the $24 \mathrm{~h}$ prior to admission to the hospital.

Rebleeding was defined as a separate episode of vomiting of fresh blood or melena, or nasogastric evidence of new bleeding after admission within the hospital or within 60 days post-admission.

Encephalopathy was defined as having symptoms and signs consistent with central nervous system involvement.

Chronic liver disease was defined by clinical criteria of stigmata of chronic liver disease with evidence of small liver span by clinical examination or ultrasound.

Renal insufficiency was defined as having acute kidney injury and/or chronic kidney disease from any cause.

All patients were treated according to standard treatment protocols of the hospital.

Data was analyzed using SPSS 23 for analysis. Relevant frequencies and tables were generated for all variables. Proportions and medians/interquartile ranges were calculated for appropriate variables. The differences in median values of continuous variables between the outcome 
groups was determined statistically by use of MannWhitney $U$ test. Incidence of mortality, rebleeding and readmission were calculated. All risk factors were analyzed to determine their association with mortality by use of the chi-square test. Relative Risk was used as a measure of association for factors associated with 60-day mortality. Cox-regression analysis was done to find out the independent risk of each categorical variable towards 60-day mortality. All factors with a $p$ value of $<0.05$ in the bivariate analysis were included in the regression model. Missing indicator variable method was used to retain cases with missing data in the regression model. Statistical significance was set at $p$ value $<0.05$.

\section{Results}

\section{Etiology of upper Gl bleeding}

From the 170 study participants, 86 underwent endoscopy. The most common cause of UGIB in these patients was oesophageal varices $(57.0 \%)$, followed by peptic ulcer disease (18.6\%) and gastritis (10.4\%) (See Fig. 1).

Patients who did not undergo endoscopy were more likely to have renal insufficiency (OR 3.30, 95\% CI 1.318.33, p 0.009) and encephalopathy (OR 4.17, 95\% CI $1.12-15.53$, p 0.023 ) on admission. None of the patients with HIV infection underwent endoscopy (p 0.006).

\section{Outcomes}

Over a period of 60 days, 57 patients died (33.5\%). Almost a quarter $(24.6 \%)$ died in the first $24 \mathrm{~h}$ after admission, and almost half (49.1\%) died within $72 \mathrm{~h}$ of admissions. Majority (96.5\%) died in the first 30 days post-admission.
Rebleeding was present in 12 patients (7.1\%). A quarter $(25.0 \%)$ rebled within the first $96 \mathrm{~h}$ of admission.

Four patients were readmitted due to UGIB in the follow up period. All of them were readmitted after 30 days and before 60 days post-admission.

Figures 2, 3, 4 and 5 show the Kaplan-Meier survival curves for the independent predictors of 60-day mortality. (See Figs. 2, 3, 4 and 5).

Patient population is described in Table 1. The association of mortality to different continuous variables (Table 2) and categorical variables (Table 3) revealed multiple significant associations. Factors that were significantly associated with mortality in univariate analysis $(\mathrm{p}<0.05)$ were entered into the regression model for multivariate analysis as shown in Table 4 .

\section{Discussion}

This prospective, cohort study aimed at identifying the major causes of upper gastrointestinal bleeding and the magnitude of mortality, rebleeding and readmission and their risk factors in a tertiary level hospital in Tanzania.

The most common cause of upper GI bleeding in this study group was oesophageal varices, found in $57 \%$ of patients. PUD accounted for $18 \%$ of cases (equally distributed among duodenal and gastric ulcer). The high prevalence of oesophageal varices has also been found in studies done in other regions of Tanzania such as Moshi [5] and Mwanza [6, 7]. In contrast, PUD was the most common cause of UGIB in studies done in Korea [8], USA [9] and various European countries [3, 10].

Oesophageal varices are most often a result of portal hypertension. Schistosomiasis and chronic liver disease are among the most common causes of portal hypertension in

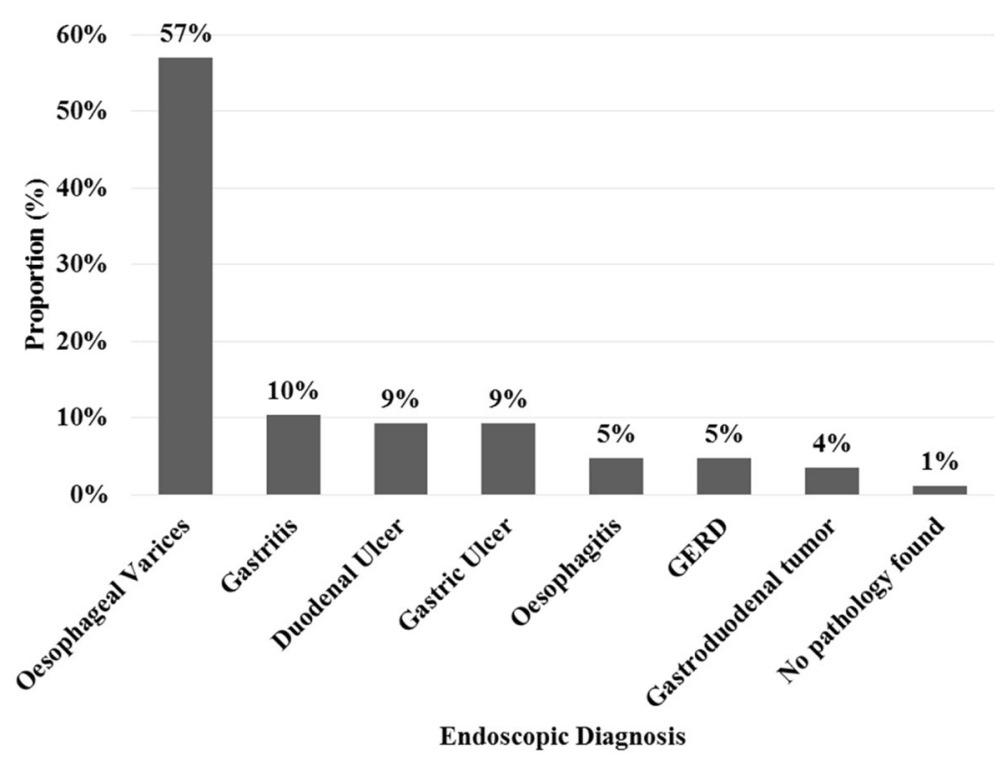

Fig. 1 Endoscopic etiologies of UGIB among 86 patients who underwent endoscopy 


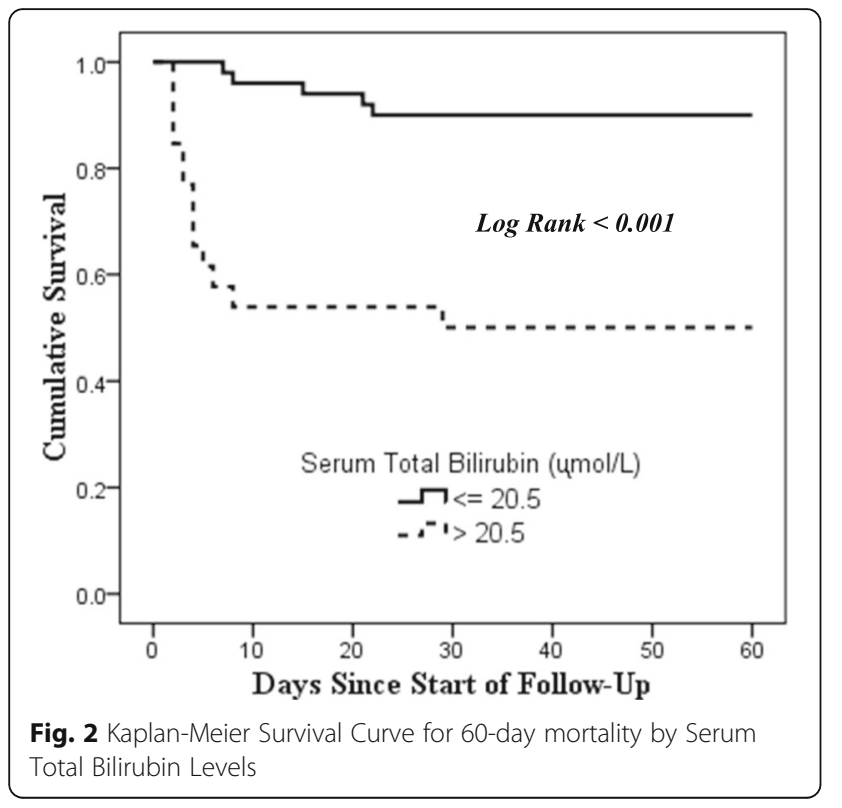

sub-Saharan Africa. The prevalence of chronic liver disease is similar to studies done in Romania [3, 4]. However, this prevalence is higher than those found in studies in Mwanza [6, 7], Europe [11] and Korea [8, 12]. One reason for this could be the type of patients involved in this study. The study was conducted in a tertiary referral center, hence it admits patients with the most severe forms of disease. Secondly, although not directly tested, the high prevalence of schistosomiasis and chronic hepatitis B infection in this setting may have contributed to the high prevalence of chronic liver disease. More than $90 \%$ of cases of schistosomiasis occur in sub-Saharan Africa and Tanzania is the

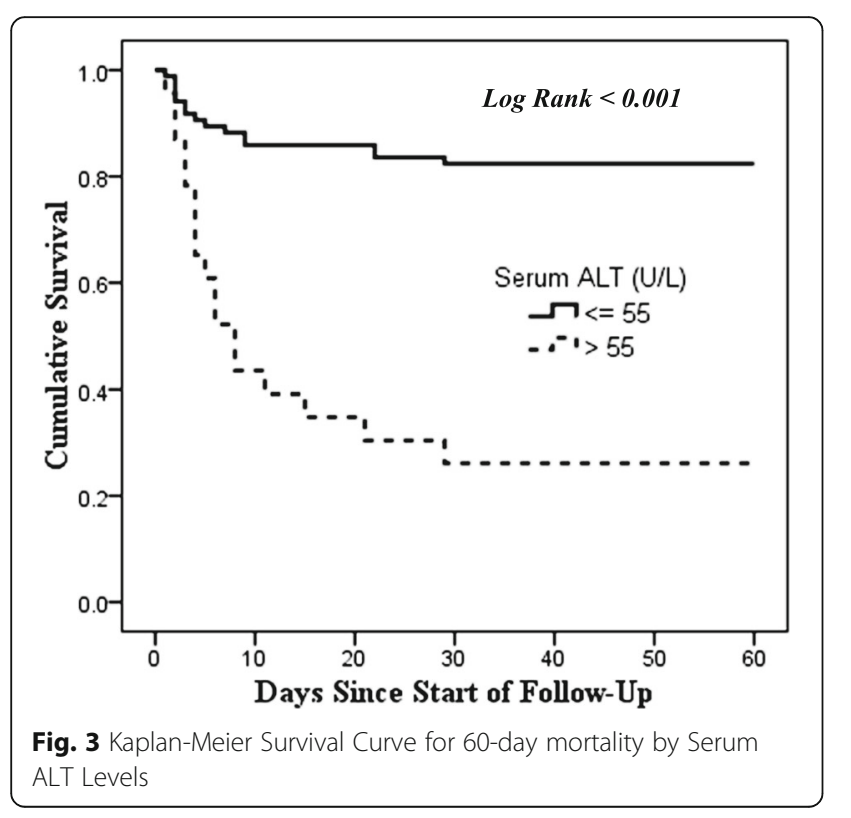

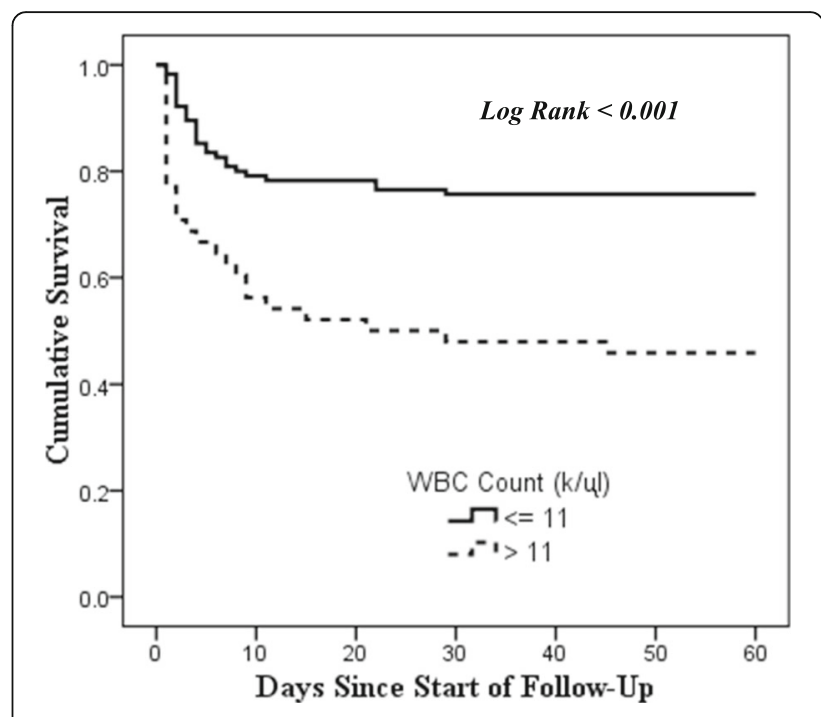

Fig. 4 Kaplan-Meier Survival Curve for 60-day mortality by WBC Count

second highest in terms of burden of disease in this region [13]. The estimated country prevalence of schistosomiasis is $51.5 \%$ [13]. One study done in Mwanza showed a high rate of active schistosome infection in adults presenting with hematemesis [7]. Similarly, one study found a $12.4 \%$ positivity of S. mansoni in stool samples [14] Studies in Tanzania have shown a prevalence of chronic hepatitis B infection ranging from 2.9-7.0\% [15-17].

PUD was the second most common diagnosis. It is well known that PUD is associated with $H$. pylori infection. Studies done in Tanzania have shown a prevalence of $H$. pylori to be more than $85 \%$ in the population [18-20]. The prevalence was shown to increase with age [19].

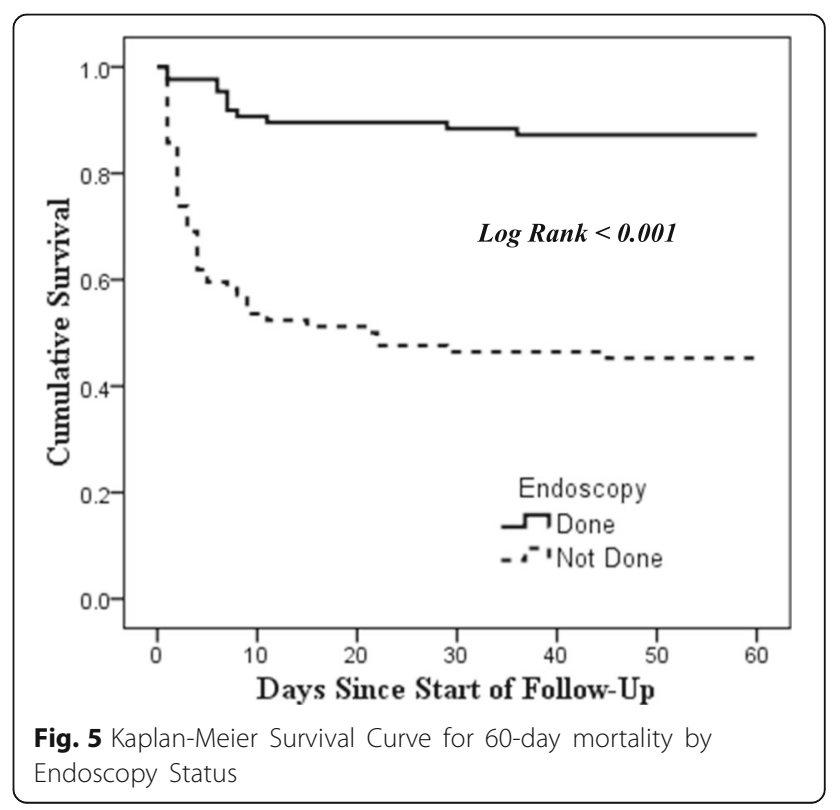


Table 1 Clinical and Demographic Characteristics of Study Population

\begin{tabular}{|c|c|}
\hline Characteristic & Median (25-75) / Frequency \\
\hline Age & $40.0(31.0-56.3)$ \\
\hline \multicolumn{2}{|l|}{ Sex } \\
\hline Male & $121(71.2 \%)$ \\
\hline Female & $49(28.8 \%)$ \\
\hline History of previous UGIB & $65(38.2 \%)$ \\
\hline Use of NSAIDs in previous one week & $7(4.1 \%)$ \\
\hline \multicolumn{2}{|l|}{ Comorbidity } \\
\hline Any Comorbidity & $106(62.4 \%)$ \\
\hline Cardiac Disease & $14(8.2 \%)$ \\
\hline Renal Insufficiency & $26(15.3 \%)$ \\
\hline Chronic Liver Disease & $52(30.6 \%)$ \\
\hline Encephalopathy & $14(8.2 \%)$ \\
\hline Malignancy & $16(9.4 \%)$ \\
\hline Hypertension & $13(7.6 \%)$ \\
\hline Diabetes Mellitus & $5(2.9 \%)$ \\
\hline HIV & $7(4.1 \%)$ \\
\hline Smoking & $9(5.3 \%)$ \\
\hline Alcohol use in previous one week & $22(12.9 \%)$ \\
\hline \multicolumn{2}{|l|}{ Initial Vital Signs } \\
\hline Systolic Blood Pressure (mmHg) & 113.5 (102.0-130.0) \\
\hline Diastolic Blood Pressure (mmHg) & $63.0(56.8-80.3)$ \\
\hline Pulse Rate (beats/min) & $101.5(86.0-108.3)$ \\
\hline Respiratory Rate (breaths/min) & $20.0(19.0-23.0)$ \\
\hline Arterial Oxygen Saturation (\%) & $100.0(100.0-100.0)$ \\
\hline \multicolumn{2}{|l|}{ Initial Laboratory Data } \\
\hline White Blood Count $(k / \mu l)$ & $8.1(4.6-12.4)$ \\
\hline Hemoglobin (g/dL) & $6.2(4.5-8.8)$ \\
\hline Platelets $(k / \mu l)$ & $178.5(90.8-293.3)$ \\
\hline Serum Creatinine $(\mu \mathrm{mol} / \mathrm{L})$ & $78.0(63.5-150.5)$ \\
\hline Serum BUN (g/dL) & $6.5(3.3-12.6)$ \\
\hline Serum ALT (U/L) & $23.0(15.0-47.0)$ \\
\hline Serum AST (U/L) & $36.0(22.0-93.0)$ \\
\hline Serum Total Bilirubin $(\mu \mathrm{mol} / \mathrm{L})$ & $12.7(7.6-29.9)$ \\
\hline Prothrombin Time (seconds) & $12.5(11.2-14.1)$ \\
\hline APTT (seconds) & $28.4(25.2-32.8)$ \\
\hline INR & $1.1(1.0-1.3)$ \\
\hline \multicolumn{2}{|l|}{ Rockall Score } \\
\hline Pre-Endoscopy & $3.0(1.0-4.0)$ \\
\hline Post-Endoscopy & $6.0(4.0-7.0)$ \\
\hline
\end{tabular}

A total 170 individuals were recruited in the study. The mean and median age of the study population was 44.9 and 40.0 years, respectively. Majority were males $(71.2 \%)$ and $38.2 \%$ had a previous episode of UGIB (either hematemesis or melena). Chronic liver disease was the most common comorbidity present (30.6\%). (See Table 1)
The overall mortality rate within 60 days of admission was $33.5 \%$. This is very high compared to the overall mortality rate from upper GI bleeding worldwide [2]. Many studies looking at mortality rates among patients with upper GI bleeding have shown mortality rates ranging from 1 to $20 \%[3,6,7,9,21-24]$. A few studies have mortality rates higher than $30 \%[10,25,26]$.

The high mortality rate in this study could be due to delayed or more severe presentation to a referral center, thus increasing the likelihood of mortality due to delay or failure in achieving hemodynamic stability, particularly in the absence of urgent endoscopic intervention. Furthermore, there is a general lack of available intervention measures such as blood transfusion and urgent endoscopic intervention and this may have contributed to the high mortality. It is noteworthy that almost half of the patients died within $72 \mathrm{~h}$ of admission to the hospital, suggesting the importance of early intervention as the key in the management of patients with acute UGIB.

A high WBC count was associated with almost 2.5 times higher rate of death compared to patients with a normal WBC count on admission. A high WBC has been associated with mortality in other studies too [27-29]. Leukocytosis is a marker for inflammation and infection, but it also occurs in other situations like trauma, exercise, drug therapy with steroids, malignancy, poisoning, psychosis and diabetic ketoacidosis. Circulating catecholamines may also lead to leukocytosis. It has been speculated that leukocytosis may represent an acute phase marker [30]. One study on upper GI bleeding patients revealed that a high WBC count was associated with more severe presentation and disease course, although it was not associated with a higher risk of mortality. The authors used a lower cut-off value of high WBC $(8.5 \mathrm{k} / \mu \mathrm{l})$ [31] Several studies have shown the association between leukocytosis and higher risk of mortality in various conditions [32-34]. It is possible that some underlying infection or inflammatory condition contributed to the mortality in these patients.

Patients admitted with elevated ALT had about 4 times higher rate of death within 60 days compared to patients admitted with normal levels of ALT. Abnormal liver enzymes, particularly a raised ALT has been shown to be a risk factor for mortality in different studies [3, 24, 35, 36].

ALT levels can be raised in patients with myocardial infarction and with ischemia to the liver [37]. Although these were not measured directly, it is possible that more severe bleeding was associated with ischemia to the heart and liver, thus contributing to the higher mortality. Higher levels of ALT have also been associated with obesity, serum cholesterol and underlying unrecognized liver diseases [37, 38]. It may be possible that these 
Table 2 Demographic and Clinical Predictors for 60-day Mortality (Continuous Variables)

\begin{tabular}{|c|c|c|c|}
\hline \multirow[t]{2}{*}{ Factor } & \multicolumn{2}{|l|}{ 60-day Mortality } & \multirow[t]{2}{*}{$p$ value } \\
\hline & $\begin{array}{l}\text { Yes } \\
\text { (Median, 25-75) }\end{array}$ & $\begin{array}{l}\text { No } \\
\text { (Median, 25-75) }\end{array}$ & \\
\hline Age & $45.0(33.5-57.5)$ & $39.0(31.0-55.5)$ & 0.168 \\
\hline \multicolumn{4}{|l|}{ Initial Vital Signs } \\
\hline Systolic Blood Pressure (mmHg) & $114.0(99.5-131.0)$ & $116.0(103.0-130.0)$ & 0.513 \\
\hline Diastolic Blood Pressure (mmHg) & $70.0(57.5-82.5)$ & $67.0(56.0-79.0)$ & 0.419 \\
\hline Pulse Rate (beats/min) & $101.0(91.0-113.0)$ & $97.0(83.5-108.0)$ & 0.066 \\
\hline Respiratory Rate (breaths/min) & $20.0(19.0-23.0)$ & $20.0(19.5-23.0)$ & 0.972 \\
\hline Arterial Oxygen Saturation (\%) & $100.0(100.0-100.0)$ & $100.0(100.0-100.0)$ & 0.035 \\
\hline \multicolumn{4}{|l|}{ Initial Laboratory Data } \\
\hline 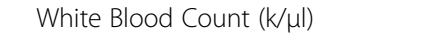 & $10.9(7.7-16.7)$ & $6.7(4.0-10.2)$ & $<0.001$ \\
\hline Hemoglobin (g/dL) & $7.4(4.9-9.3)$ & $6.0(4.4-8.2)$ & 0.058 \\
\hline Platelets $(k / \mu l)$ & $183.0(133.0-278.0)$ & $172.5(77.4-300.3)$ & 0.339 \\
\hline Serum Creatinine $(\mu \mathrm{mol} / \mathrm{L})$ & $105.5(72.3-289.2)$ & $69.4(62.2-101.6)$ & 0.001 \\
\hline Serum BUN (g/dL) & $9.7(4.7-23.4)$ & $4.5(3.0-9.1)$ & 0.001 \\
\hline Serum ALT (U/L) & $58.0(14.3-113.0)$ & $21.0(15.0-35.0)$ & 0.004 \\
\hline Serum AST (U/L) & $74.0(33.5-274.0)$ & $29.5(18.0-50.8)$ & $<0.001$ \\
\hline Serum Total Bilirubin $(\mu \mathrm{mol} / \mathrm{L})$ & $83.5(17.7-421.1)$ & $11.3(7.0-17.6)$ & 0.001 \\
\hline Prothrombin Time (seconds) & $14.1(12.7-19.4)$ & $12.1(11.0-13.3)$ & 0.002 \\
\hline APTT (seconds) & $28.5(24.9-37.5)$ & $28.4(25.2-31.4)$ & 0.533 \\
\hline INR & $1.3(1.2-1.7)$ & $1.1(1.0-1.2)$ & 0.001 \\
\hline \multicolumn{4}{|l|}{ Rockall Score } \\
\hline Pre-Endoscopy Rockall Score & $3.0(2.0-4.0)$ & $3.0(1.0-4.0)$ & 0.143 \\
\hline Post-Endoscopy Rockall Score & $6.0(5.0-7.0)$ & $6.0(4.0-7.0)$ & 0.620 \\
\hline
\end{tabular}

Comparison of medians of different continuous variables revealed that the following were all associated with a higher risk of mortality: A raised WBC, serum creatinine, serum BUN, serum ALT, serum AST, serum total bilirubin, prothrombin time and INR and reduced oxygen saturation on admission $(p<0.05)$. (See Table 2)

comorbidities may have contributed to mortality in this group of patients.

A high serum total bilirubin during admission was associated with an almost 6 times increased rate of death in this study population. Other studies have also shown this association between high serum total bilirubin and mortality $[21,22,25,31,36,39,40]$. High levels of serum total bilirubin have been associated with all-cause mortality [41, 42]. The exact mechanism is unclear. It has been shown in different studies that a high level of serum total bilirubin is associated with cardiovascular disease, particularly ischemic heart disease [43] and acute myocardial infarction [44]. The increased risk of acute cardiovascular events in patients with high serum total bilirubin may have contributed to the increased mortality rate in this study group.

Patients who did not undergo endoscopy during their hospital stay had about 4 times a higher rate of death within 60 days compared to patients who had an endoscopy done. Lack of endoscopy was shown to be a risk factor for mortality in another study done in Bugando [7]. It may be possible that patients who were hemodynamically unstable and very sick overall on admission did not undergo endoscopy and were at higher risk of mortality, as seen by the fact that patients with renal insufficiency, HIV and encephalopathy were all less likely to undergo endoscopy. Consensus guidelines outline the importance of endoscopy in acute upper GI bleeding. It helps to identify the source of bleeding, provide prognostic information regarding the risk of bleeding, and offer therapy for hemostasis [45]. One study also showed reduced incidence of myocardial infarction and overall mortality in patients who received initial resuscitation (including endoscopic intervention) [46] Hence, the patients who did not undergo endoscopy may have missed the chance to be offered endoscopic intervention because of a lack of diagnosis and this may be the reason for the increased mortality rate in this group of patients.

The strength of this study was that it was a prospective study where many variables that could potentially predict adverse outcomes were assessed. Furthermore, all patients were followed-up in this study. 
Table 3 Demographic and Clinical Predictors for 60-day Mortality (Categorical Variables)

\begin{tabular}{|c|c|c|c|c|c|}
\hline \multirow[t]{2}{*}{ Factor } & \multicolumn{3}{|c|}{ 60-day Mortality } & \multirow{2}{*}{$\begin{array}{l}\text { Relative Risk } \\
(95 \% \mathrm{Cl})\end{array}$} & \multirow[t]{2}{*}{$P$ value } \\
\hline & Yes & No & Total & & \\
\hline \multicolumn{6}{|l|}{ Age } \\
\hline$>40$ years & $31(37.3 \%)$ & $52(62.7 \%)$ & 83 & 1.25 & \multirow[t]{2}{*}{0.303} \\
\hline$\leq 40$ years & $26(29.9 \%)$ & $61(70.1 \%)$ & 87 & $(0.82-1.91)$ & \\
\hline \multicolumn{6}{|l|}{ Sex } \\
\hline Male & $41(33.9 \%)$ & $80(66.1 \%)$ & 121 & 1.04 & \multirow[t]{2}{*}{0.878} \\
\hline Female & $16(32.7 \%)$ & $33(67.3 \%)$ & 49 & $(0.65-1.67)$ & \\
\hline \multicolumn{6}{|c|}{ History of previous UGIB } \\
\hline Yes & $21(27.6 \%)$ & $55(72.4 \%)$ & 76 & 0.72 & \multirow[t]{2}{*}{0.143} \\
\hline No & $36(38.3 \%)$ & $58(61.7 \%)$ & 94 & $(0.46-1.13)$ & \\
\hline \multicolumn{6}{|c|}{ Use of NSAIDs in previous one week } \\
\hline Yes & $1(14.3 \%)$ & $6(85.7 \%)$ & 7 & 0.42 & \multirow[t]{2}{*}{0.426} \\
\hline No & $56(34.4 \%)$ & 107 (65.6\%) & 163 & $(0.07-2.58)$ & \\
\hline \multicolumn{6}{|c|}{ Any Comorbidity } \\
\hline Yes & $40(37.7 \%)$ & $66(62.3 \%)$ & 106 & 1.42 & \multirow[t]{2}{*}{0.135} \\
\hline No & $17(26.6 \%)$ & 47 (73.4\%) & 64 & $(0.88-2.29)$ & \\
\hline \multicolumn{6}{|c|}{ Cardiac Disease } \\
\hline Yes & $6(42.9 \%)$ & $8(57.1 \%)$ & 14 & 1.31 & \multirow[t]{2}{*}{0.556} \\
\hline No & $51(32.7 \%)$ & 105 (67.3\%) & 156 & $(0.69-2.50)$ & \\
\hline \multicolumn{6}{|c|}{ Renal Insufficiency } \\
\hline Yes & $16(61.5 \%)$ & $10(38.5 \%)$ & 26 & 2.16 & \multirow[t]{2}{*}{0.001} \\
\hline No & $41(28.5 \%)$ & 103 (71.5\%) & 144 & $(1.45-3.22)$ & \\
\hline \multicolumn{6}{|c|}{ Chronic Liver Disease } \\
\hline Yes & $21(28.0 \%)$ & $54(72.0 \%)$ & 75 & 0.74 & \multirow[t]{2}{*}{0.175} \\
\hline No & $36(37.9 \%)$ & $59(62.1 \%)$ & 95 & $(0.47-1.15)$ & \\
\hline \multicolumn{6}{|c|}{ Encephalopathy } \\
\hline Yes & $11(78.6 \%)$ & $3(21.4 \%)$ & 14 & 2.67 & \multirow[t]{2}{*}{$<0.001$} \\
\hline No & $46(29.5 \%)$ & 110 (70.5\%) & 156 & $(1.85-3.84)$ & \\
\hline \multicolumn{6}{|l|}{ Malignancy } \\
\hline Yes & $9(56.3 \%)$ & 7 (43.8\%) & 16 & 1.81 & \multirow[t]{2}{*}{0.043} \\
\hline No & $48(31.2 \%)$ & 106 (68.8\%) & 154 & $(1.1-2.95)$ & \\
\hline Hypertension & & & & & \\
\hline Yes & $6(46.2 \%)$ & 7 (53.8\%) & 13 & 1.42 & 0.364 \\
\hline No & $51(32.5 \%)$ & 106 (67.5\%) & 157 & $(0.76-2.67)$ & \\
\hline Diabetes Melli & & & & & \\
\hline Yes & $4(80.0 \%)$ & $1(20.0 \%)$ & 5 & 2.49 & 0.044 \\
\hline No & $53(32.1 \%)$ & 112 (67.9\%) & 165 & $(1.52-4.07)$ & \\
\hline HIV & & & & & \\
\hline Yes & $5(71.4 \%)$ & $2(28.6 \%)$ & 7 & 2.24 & 0.043 \\
\hline No & 52 (31.9\%) & 111 (68.1\%) & 163 & $(1.33-3.76)$ & \\
\hline Smoking & & & & & \\
\hline Yes & $3(33.3 \%)$ & $6(66.7 \%)$ & 9 & 0.99 & 1.000 \\
\hline No & $54(33.5 \%)$ & 107 (66.5\%) & 161 & $(0.39-2.57)$ & \\
\hline
\end{tabular}


Table 3 Demographic and Clinical Predictors for 60-day Mortality (Categorical Variables) (Continued)

\begin{tabular}{|c|c|c|c|c|c|}
\hline \multirow[t]{2}{*}{ Factor } & \multicolumn{3}{|c|}{ 60-day Mortality } & \multirow{2}{*}{$\begin{array}{l}\text { Relative Risk } \\
(95 \% \mathrm{Cl})\end{array}$} & \multirow[t]{2}{*}{$P$ value } \\
\hline & Yes & No & Total & & \\
\hline \multicolumn{6}{|c|}{ Alcohol use in previous one week } \\
\hline Yes & $8(36.4 \%)$ & $14(63.6 \%)$ & 22 & 1.10 & \multirow[t]{2}{*}{0.763} \\
\hline No & $49(33.1 \%)$ & $99(66.9 \%)$ & 148 & $(0.60-2.00)$ & \\
\hline \multicolumn{6}{|c|}{ Systolic Blood Pressure } \\
\hline$<110 \mathrm{mmHg}$ & $23(37.1 \%)$ & $39(62.9 \%)$ & 62 & 1.18 & \multirow[t]{2}{*}{0.455} \\
\hline$\geq 110 \mathrm{mmHg}$ & $34(31.5 \%)$ & $74(68.5 \%)$ & 108 & $(0.77-1.81)$ & \\
\hline \multicolumn{6}{|c|}{ Diastolic Blood Pressure } \\
\hline$<60 \mathrm{mmHg}$ & $17(31.5 \%)$ & $37(68.5 \%)$ & 54 & 0.91 & \multirow[t]{2}{*}{0.700} \\
\hline$\geq 60 \mathrm{mmHg}$ & $40(34.5 \%)$ & $76(65.5 \%)$ & 116 & $(0.57-1.46)$ & \\
\hline \multicolumn{6}{|l|}{ Pulse Rate } \\
\hline$>100$ bpm & $30(38.5 \%)$ & $48(61.5 \%)$ & 78 & 1.31 & \multirow[t]{2}{*}{0.210} \\
\hline$\leq 100 \mathrm{bpm}$ & $27(29.3 \%)$ & $65(70.7 \%)$ & 92 & $(0.86-2.00)$ & \\
\hline \multicolumn{6}{|l|}{ Respiratory Rate } \\
\hline$>18 \mathrm{bpm}$ & $44(32.6 \%)$ & $91(67.4 \%)$ & 135 & 0.88 & \multirow[t]{2}{*}{0.611} \\
\hline$\leq 18 \mathrm{bpm}$ & $13(37.1 \%)$ & $22(62.9 \%)$ & 35 & $(0.54-1.44)$ & \\
\hline \multicolumn{6}{|c|}{ Arterial Oxygen Saturation } \\
\hline$<92 \%$ & $2(50.0 \%)$ & $2(50.0 \%)$ & 4 & 1.51 & \multirow[t]{2}{*}{0.603} \\
\hline$\geq 92 \%$ & $55(33.1 \%)$ & $111(66.9 \%)$ & 166 & $(0.55-4.12)$ & \\
\hline \multicolumn{6}{|c|}{ White Blood Count $(\mathrm{k} / \mu \mathrm{l})$} \\
\hline$>11.00$ & $26(54.2 \%)$ & $22(45.8 \%)$ & 48 & 2.23 & \multirow[t]{2}{*}{$<0.001$} \\
\hline$\leq 11.00$ & $28(24.3 \%)$ & 87 (75.7\%) & 115 & $(1.47-3.37)$ & \\
\hline \multicolumn{6}{|c|}{ Hemoglobin (g/dL) } \\
\hline$<10.00$ & $45(31.7 \%)$ & $97(68.3 \%)$ & 142 & 0.74 & \multirow[t]{2}{*}{0.310} \\
\hline$\geq 10.00$ & $9(42.9 \%)$ & $12(57.1 \%)$ & 21 & $(0.43-1.28)$ & \\
\hline \multicolumn{6}{|l|}{ Platelets $(\mathrm{k} / \mu \mathrm{l})$} \\
\hline$<140$ & $18(26.5 \%)$ & $50(73.5 \%)$ & 68 & 0.69 & \multirow[t]{2}{*}{0.115} \\
\hline$\geq 140$ & $36(38.3 \%)$ & $58(61.7 \%)$ & 94 & $(0.43-1.11)$ & \\
\hline \multicolumn{6}{|c|}{ Serum Creatinine $(\mu \mathrm{mol} / \mathrm{L})$} \\
\hline$>115$ & $21(53.8 \%)$ & $18(46.2 \%)$ & 39 & 2.20 & \multirow[t]{2}{*}{0.001} \\
\hline$\leq 115$ & $22(24.4 \%)$ & $68(75.6 \%)$ & 90 & $(1.38-3.51)$ & \\
\hline Serum BUN $(g / d l$ & & & & & \\
\hline$>7.4$ & $21(47.7 \%)$ & $23(52.3 \%)$ & 44 & 2.39 & 0.003 \\
\hline$\leq 7.4$ & $12(20.0 \%)$ & $48(80.0 \%)$ & 60 & $(1.32-4.32)$ & \\
\hline Serum ALT (U/L) & & & & & \\
\hline$>55$ & 17 (73.9\%) & $6(26.1 \%)$ & 23 & 4.19 & $<0.001$ \\
\hline$\leq 55$ & $15(17.6 \%)$ & 70 (82.4\%) & 85 & $(2.49-7.04)$ & \\
\hline Serum AST (U/L) & & & & & \\
\hline$>34$ & $24(40.7 \%)$ & 35 (59.3\%) & 59 & 2.35 & 0.007 \\
\hline$\leq 34$ & 9 (17.3\%) & $43(82.7 \%)$ & 52 & $(1.20-4.59)$ & \\
\hline Serum Total Biliru & & & & & \\
\hline$>20.5$ & $13(50.0 \%)$ & $13(50.0 \%)$ & 26 & 5.00 & $<0.001$ \\
\hline$\leq 20.5$ & $5(10.0 \%)$ & 45 (90.0\%) & 50 & $(2.0-12.50)$ & \\
\hline
\end{tabular}


Table 3 Demographic and Clinical Predictors for 60-day Mortality (Categorical Variables) (Continued)

\begin{tabular}{|c|c|c|c|c|c|}
\hline \multirow[t]{2}{*}{ Factor } & \multicolumn{3}{|c|}{ 60-day Mortality } & \multirow{2}{*}{$\begin{array}{l}\text { Relative Risk } \\
(95 \% \mathrm{Cl})\end{array}$} & \multirow[t]{2}{*}{$P$ value } \\
\hline & Yes & No & Total & & \\
\hline \multicolumn{6}{|l|}{ Prothrombin Time (seconds) } \\
\hline$>12.1$ & $13(46.4 \%)$ & $15(53.6 \%)$ & 28 & 3.71 & \multirow[t]{2}{*}{0.008} \\
\hline$\leq 12.1$ & $3(12.5 \%)$ & $21(87.5 \%)$ & 24 & $(1.20-11.51)$ & \\
\hline \multicolumn{6}{|l|}{ APTT (seconds) } \\
\hline$>30.4$ & 7 (35.0\%) & $13(65.0 \%)$ & 20 & 1.08 & \multirow[t]{2}{*}{0.842} \\
\hline$\leq 30.4$ & $11(32.4 \%)$ & $23(67.6 \%)$ & 34 & $(0.50-2.34)$ & \\
\hline \multicolumn{6}{|l|}{ INR } \\
\hline$>1.13$ & $13(48.1 \%)$ & $14(51.9 \%)$ & 27 & 4.17 & \multirow[t]{2}{*}{0.004} \\
\hline$\leq 1.13$ & $3(11.5 \%)$ & $23(88.5 \%)$ & 26 & $(1.34-12.97)$ & \\
\hline \multicolumn{6}{|l|}{ Endoscopy } \\
\hline Not Done & $46(54.8 \%)$ & $38(45.2 \%)$ & 84 & 4.28 & \multirow[t]{2}{*}{$<0.001$} \\
\hline Done & $11(12.8 \%)$ & $75(87.2 \%)$ & 86 & $(2.39-7.69)$ & \\
\hline \multicolumn{6}{|l|}{ Endoscopy Diagnosis } \\
\hline Oesophageal Varices & $6(12.2 \%)$ & $43(87.8 \%)$ & 49 & 0.91 & \multirow[t]{2}{*}{1.000} \\
\hline Other Diagnosis & $5(13.5 \%)$ & $32(86.5 \%)$ & 37 & $(0.30-2.74)$ & \\
\hline \multicolumn{4}{|l|}{ Pre-Endoscopy Rockall Score } & 1.29 & \\
\hline$>3$ & $21(39.6 \%)$ & $32(60.4 \%)$ & 53 & $(0.84-1.98)$ & \multirow[t]{2}{*}{0.257} \\
\hline$\leq 3$ & $36(30.8 \%)$ & $81(69.2 \%)$ & 117 & & \\
\hline \multicolumn{6}{|l|}{ Post-Endoscopy Rockall Score } \\
\hline$>5$ & $8(15.1 \%)$ & $45(84.9 \%)$ & 53 & 1.61 & \multirow[t]{2}{*}{0.524} \\
\hline$\leq 5$ & $3(9.4 \%)$ & $29(90.6 \%)$ & 32 & $(0.46-5.63)$ & \\
\hline
\end{tabular}

Mortality was also compared to different categorical variables as outlined in Table 3. A high serum total bilirubin was associated with a 5 -fold increased risk of mortality $(\mathrm{RR}=5.00, p<0.001)$. Patients with a raised serum ALT level $(\mathrm{RR}=4.19, p<0.001)$, a raised INR $(\mathrm{RR}=4.17, p=0.004)$, a prolonged prothrombin time ( $R R=3.71, p=0.008)$ and those who did not undergo endoscopy during admission ( $R R=4.28, p<0.001)$ had an almost 4-fold increased risk of mortality within 60 days from admission. Other factors that were associated with a higher risk of mortality included patients with renal insufficiency (RR $=2.16, p<0.001)$, encephalopathy $(\mathrm{RR}=2.67, p<0.001)$, malignancy $(\mathrm{RR}=1.81, p=0.043)$, diabetes mellitus $(\mathrm{RR}=2.49, p=0.044)$, HIV disease $(\mathrm{RR}=2.24, p=0.043)$, a raised WBC count $(\mathrm{RR}=2.23, p<0.001)$, a raised serum creatinine level $(\mathrm{RR}=2.20, p=0.001)$, a raised serum $\mathrm{BUN}$ level $(\mathrm{RR}=2.39, p=0.003)$, and a raised serum $\mathrm{AST}$ level (RR $=2.35, p=0.007)$. (See Table 3)

The study had some limitations. Half of the patients did not undergo endoscopy due to not being able to afford it. This may have underestimated the burden of some etiologies. Importantly, not undergoing endoscopy was an independent predictor of mortality. Therefore, more serious etiologies may have been missed which led to early death. Some laboratory values were not obtained for some patients and this may have underestimated or overestimated the significance of these factors with the outcomes. Presence of comorbidities was assessed by patient interview or clinical diagnosis. True melena was ascertained verbally only. This may have included some patients who had black stool from ingestion of previously prescribed ferrous sulphate. Gold standard criteria for diagnosis of comorbidities were not used in all patients. This study identified an important area in the local country but the results may not necessarily be generalisable to settings with different epidemiological disease patterns, particularly for chronic liver disease. Subgroup analysis to identify specific risk factors for mortality following variceal bleeding and ulcer bleeding could not be done due to small sample size these subgroups would have.

\section{Conclusions}

Oesophageal varices was the most common cause of UGIB, followed by peptic ulcer disease. There was a high burden of mortality in this study, about onethird of patients admitted with UGIB died within 60 days. Rebleeding and readmission rates were low. Independent predictors of 60-day mortality were a high WBC count, raised serum ALT, raised serum total bilirubin and a lack of endoscopy. It is recommended that patients require early and more aggressive intervention in acute UGIB and possibly larger studies can be conducted looking at other factors that 
Table 4 Cox-Regression Analysis of Predictors for 60-day Mortality (Multivariate Analysis)

\begin{tabular}{lll}
\hline Predictor & Hazard Ratio $(95 \% \mathrm{Cl})$ & $P$ value \\
\hline Renal Insufficiency & $1.38(0.54-3.51)$ & 0.504 \\
Encephalopathy & $1.19(0.46-3.06)$ & 0.723 \\
HIV & $0.58(0.16-2.07)$ & 0.402 \\
Diabetes Mellitus & $2.14(0.54-8.50)$ & 0.279 \\
Malignancy & $0.58(0.21-1.64)$ & 0.303 \\
White Blood Cell Count $>11 \mathrm{k} / \mu \mathrm{l}$ & $2.45(1.23-4.89)$ & 0.011 \\
Serum Creatinine $>115 \mu \mathrm{mol} / \mathrm{L}$ & $1.55(0.57-4.23)$ & 0.896 \\
Serum BUN $>7.4 \mathrm{~g} / \mathrm{dL}$ & $1.46(0.55-3.85)$ & 0.445 \\
Serum ALT >55 U/L & $4.22(1.31-13.57)$ & 0.016 \\
Serum AST > 34 U/L & $1.11(0.39-3.17)$ & 0.843 \\
Serum Total Bilirubin > 20.5 $\mu \mathrm{mol} / \mathrm{L}$ & $5.79(1.58-21.25)$ & 0.008 \\
Prothrombin Time > 12.1 s & $0.33(0.01-21.14)$ & 0.598 \\
INR $>1.13$ & $8.57(0.14-536.04)$ & 0.309 \\
Endoscopy Not Done & $4.40(2.11-9.17)$ & $<0.001$ \\
\hline
\end{tabular}

Multivariate analysis to identify independent predictors of 60-day mortality was done using Cox-Regression analysis. Factors that were shown to be significantly associated with a higher risk of 60-day mortality by bivariate analysis were included in the regression model. Multivariate analysis showed that a higher WBC count of $>11 \mathrm{k} / \mu \mathrm{l}$, a high serum $\mathrm{ALT}>55 \mathrm{U} / \mathrm{L}$, a high serum total bilirubin $>20.5 \mu \mathrm{mol} / \mathrm{L}$ were independently associated with an increased risk of mortality. Patients who did not undergo endoscopy had a 4.4 times higher rate of death within 60 days of admission (Table 4)

may contribute to mortality in UGIB patients. It is also important to study the reasons for a lack of endoscopy among patients with UGIB since this is a potentially correctable factor which can then lead to reduction in mortality and improvement on clinical care of these patients in the country.

\section{Abbreviations}

ALT: Alanine Aminotransferase; aPTT: Activated Partial Thromboplastin Time; AST: Aspartate Aminotransferase; BUN: Blood Urea Nitrogen; HIV: Human Immunodeficiency Virus; HR: Heart Rate; INR: International Normalized Ratio; MNH: Muhimbili National Hospital; MUHAS: Muhimbili University of Health and Allied Sciences; PT: Prothrombin Time; SBP: Systolic Blood Pressure; UGIB: Upper Gastrointestinal Bleeding

\section{Acknowledgements}

To the Department of Internal Medicine at MUHAS and MNH. To Dr. Khadija M Bhimji. To the study participants.

\section{Funding}

The study received no funding.

\section{Availability of data and materials}

The data that support the findings of this study are available from the Muhimbili University of Health and Sciences but restrictions apply to the availability of these data, which were used under license for the current study, and so are not publicly available. Data are however available from the authors upon reasonable request and with permission of Muhimbili University of Health and Allied Sciences.

\section{Authors' contributions}

SM designed the study, collected, analyzed and interpreted the data and was a major contributor in writing the manuscript. EK was involved in study design and revision of the manuscript. Both authors read and approved the final manuscript.

\section{Ethics approval and consent to participate}

Permission to conduct the study was sought from relevant ethical committees at MNH and Muhimbili University of Health and Allied Sciences (MUHAS). (Ref No: MU/PGS/SAECNol. XIV). All patients were entered into the study after a written informed consent, either given by the patients themselves or their guardians in the case where patients were not able to. All patients received treatment as per standard hospital policies. The data obtained during the study was kept anonymous.

\section{Consent for publication}

Not applicable.

\section{Competing interests}

The authors declare that they have no competing interests.

\section{Publisher's Note}

Springer Nature remains neutral with regard to jurisdictional claims in published maps and institutional affiliations.

Received: 20 February 2017 Accepted: 27 November 2017

Published online: 20 December 2017

\section{References}

1. Wilkins T, Khan N, Nabh A, Schade RR. Diagnosis and management of upper gastrointestinal bleeding. Am Fam Physician. 2012;85(5):469-76.

2. Palmer K, Atkinson S, Donnelly M, Forbes-Young R, Gomez C, Greer D, Halligan K, Hauser M, McPherson S, McCord M, et al. Acute upper gastrointestinal bleeding: management. UK: National Institute for Health and Clinical Excellence.

3. Balaban DV, Strambu V, Florea BG, Cazan AR, Bratucu M, Jinga M. Predictors for in-hospital mortality and need for clinical intervention in upper Gl bleeding: a 5-year observational study. Chirurgia (Bucharest, Romania 1990). 2014;109(1):48-54

4. Matei D, Groza I, Furnea B, Puie L, Levi C, Chiru A, Cruciat C, Mester G, Vesa SC, Tantau M. Predictors of variceal or nonvariceal source of upper gastrointestinal bleeding. An etiology predictive score established and validated in a tertiary referral center. Journal of gastrointestinal and liver diseases : JGLD. 2013:22(4):379-84

5. Suba M, Ayana SM, Mtabho CM, Kibiki GS. The aetiology, management and clinical outcome of upper gastrointestinal bleeding among patients admitted at the Kilimanjaro Christian medical Centre in Moshi, Tanzania. Tanzania journal of health research. 2010;12(4):302-5.

6. Jaka H, Koy M, Liwa A, Kabangila R, Mirambo M, Scheppach W, Mkongo E, McHembe MD, Chalya PL. A fibreoptic endoscopic study of upper gastrointestinal bleeding at Bugando medical Centre in northwestern Tanzania: a retrospective review of 240 cases. BMC Res Notes. 2012;5:200.

7. Chofle AA, Jaka H, Koy M, Smart LR, Kabangila R, Ewings FM, Mazigo HD, Johnson WD Jr, Fitzgerald DW, Peck RN, et al. Oesophageal varices, schistosomiasis, and mortality among patients admitted with haematemesis in Mwanza, Tanzania: a prospective cohort study. BMC Infect Dis. 2014;14:303.

8. Lee YJ, Min BR, Kim ES, Park KS, Cho KB, Jang BK, Chung WJ, Hwang JS, Jeon SW. Predictive factors of mortality within 30 days in patients with nonvariceal upper gastrointestinal bleeding. The Korean journal of internal medicine. 2016:31(1):54-64.

9. Go JT, Vaughan-Sarrazin M, Auerbach A, Schnipper J, Wetterneck TB, Gonzalez D, Meltzer D, Kaboli PJ. Do hospitalists affect clinical outcomes and efficiency for patients with acute upper gastrointestinal hemorrhage $(U G \mid H)$ ? Journal of hospital medicine : an official publication of the society of. Hospital Medicine. 2010;5(3):133-9.

10. Skok P, Sinkovic A. Upper gastrointestinal haemorrhage: predictive factors of in-hospital mortality in patients treated in the medical intensive care unit. The Journal of international medical research. 2011;39(3):1016-27.

11. Lanas A, Aabakken L, Fonseca J, Mungan ZA, Papatheodoridis GV Piessevaux H, Cipolletta L, Nuevo J, Tafalla M. Clinical predictors of poor outcomes among patients with nonvariceal upper gastrointestinal bleeding in Europe. Aliment Pharmacol Ther. 2011;33(11):1225-33.

12. Lee YJ, Kim ES, Hah YJ, Park KS, Cho KB, Jang BK, Chung WJ, Hwang JS. Chronic kidney disease, hemodynamic instability, and endoscopic high-risk appearance are associated with 30-day rebleeding in patients with non-variceal upper gastrointestinal bleeding. J Korean Med Sci. 2013;28(10):1500-6. 
13. Mazigo HD, Nuwaha F, Kinung'hi SM, Morona D, Pinot de Moira A, Wilson S, Heukelbach J, Dunne DW. Epidemiology and control of human schistosomiasis in Tanzania. Parasites \& vectors. 2012;5:274.

14. Siza JE, Kaatano GM, Chai JY, Eom KS, Rim HJ, Yong TS, Min DY, Chang SY, Ko Y, Changalucha JM. Prevalence of Schistosomes and soil-transmitted helminths and morbidity associated with schistosomiasis among adult population in Lake Victoria Basin, Tanzania. The Korean journal of parasitology. 2015;53(5):525-33.

15. Mueller A, Stoetter L, Kalluvya S, Stich A, Majinge C, Weissbrich B, Kasang C. Prevalence of hepatitis B virus infection among health care workers in a tertiary hospital in Tanzania. BMC Infect Dis. 2015;15:386.

16. Muro FJ, Fiorillo SP, Sakasaka P, Odhiambo C, Reddy EA, Cunningham CK, Buchanan AM. Seroprevalence of hepatitis B and C viruses among children in Kilimanjaro region, Tanzania. Journal of the Pediatric Infectious Diseases Society. 2013;2(4):320-6.

17. Rashid S, Kilewo C, Aboud S. Seroprevalence of hepatitis B virus infection among antenatal clinic attendees at a tertiary hospital in Dar es salaam, Tanzania. Tanzania journal of health research. 2014;16(1):9-15.

18. Chalya PL, Mabula JB, Koy M, McHembe MD, Jaka HM, Kabangila R, Chandika AB, Gilyoma JM. Clinical profile and outcome of surgical treatment of perforated peptic ulcers in northwestern Tanzania: a tertiary hospital experience. World journal of emergency surgery : WJES. 2011;6:31.

19. Mbulaiteye SM, Gold BD, Pfeiffer RM, Brubaker GR, Shao J, Biggar RJ, Hisada M. H. Pylori-infection and antibody immune response in a rural Tanzanian population. Infectious agents and cancer. 2006;1:3.

20. Aoki K, Kihaile PE, Castro M, Disla M, Nyambo TB, Misumi J. Seroprevalences ofHelicobacter pylori infection and chronic atrophic gastritis in the united republic of Tanzania and the Dominican Republic. Environ Health Prev Med. 2004;9(4):170-5.

21. Hsu YC, Liou JM, Chung CS, Tseng CH, Lin TL, Chen CC, MS W, Wang HP. Early risk stratification with simple clinical parameters for cirrhotic patients with acute upper gastrointestinal bleeding. Am J Emerg Med. 2010;28(8):884-90.

22. Kumar AS, Sibia RS. Predictors of in-hospital mortality among patients presenting with variceal gastrointestinal bleeding. Saudi journal of gastroenterology : official journal of the Saudi Gastroenterology Association. 2015:21(1):43-6.

23. Katschinski B, Logan R, Davies J, Faulkner G, Pearson J, Langman M. Prognostic factors in upper gastrointestinal bleeding. Dig Dis Sci. 1994;39(4):706-12.

24. Zimmerman J, Siguencia J, Tsvang E, Beeri R, Arnon R. Predictors of mortality in patients admitted to hospital for acute upper gastrointestinal hemorrhage. Scand J Gastroenterol. 1995;30(4):327-31.

25. Gatta A, Merkel C, Amodio P, Bellon S, Bellumat A, Bolognesi M, Borsato L, Butto M, Casson FF, Cavallarin G, et al. Development and validation of a prognostic index predicting death after upper gastrointestinal bleeding in patients with liver cirrhosis: a multicenter study. Am J Gastroenterol. 1994; 89(9):1528-36.

26. Roberts SE, Button LA, Williams JG. Prognosis following upper gastrointestinal bleeding. PLoS One. 2012;7(12):e49507.

27. Adamopoulos AB, Baibas NM, Efstathiou SP, Tsioulos DI, Mitromaras AG, Tsami AA, Mountokalakis TD. Differentiation between patients with acute upper gastrointestinal bleeding who need early urgent upper gastrointestinal endoscopy and those who do not. A prospective study. Eur J Gastroenterol Hepatol. 2003;15(4):381-7.

28. Weng SC, Shu KH, Tarng DC, Tang YJ, Cheng CH, Chen CH, TM Y, Chuang YW, Huang ST, Sheu WH, et al. In-hospital mortality risk estimation in patients with acute nonvariceal upper gastrointestinal bleeding undergoing hemodialysis: a retrospective cohort study. Ren Fail. 2013:35(2):243-8.

29. Patch D, Nikolopoulou V, McCormick A, Dick R, Armonis A, Wannamethee G, Burroughs A. Factors related to early mortality after transjugular intrahepatic portosystemic shunt for failed endoscopic therapy in acute variceal bleeding. J Hepatol. 1998;28(3):454-60.

30. Asadollahi K, Beeching NJ, Gill GV. Leukocytosis as a predictor for noninfective mortality and morbidity. QJM. 2010;103(5):285-92.

31. Chalasani N, Patel K, Clark WS, Wilcox CM. The prevalence and significance of leukocytosis in upper gastrointestinal bleeding. Am J Med Sci. 1998; 315(4):233-6.

32. de Labry LO, Campion EW, Glynn RJ, Vokonas PS. White blood cell count as a predictor of mortality: results over 18 years from the normative aging study. J Clin Epidemiol. 1990;43(2):153-7.

33. Cannon CP, McCabe CH, Wilcox RG, Bentley JH, Braunwald E. Association of white blood cell count with increased mortality in acute myocardial infarction and unstable angina pectoris. OPUS-TIMI 16 investigators. Am J Cardiol. 2001;87(5):636-9. a610
34. Barron HV, Harr SD, Radford MJ, Wang Y, Krumholz HM. The association between white blood cell count and acute myocardial infarction mortality in patients > or $=65$ years of age: findings from the cooperative cardiovascular project. J Am Coll Cardiol. 2001;38(6):1654-61.

35. Klebl F, Bregenzer N, Schofer L, Tamme W, Langgartner J, Scholmerich J, Messmann $\mathrm{H}$. Risk factors for mortality in severe upper gastrointestinal bleeding. Int J Color Dis. 2005;20(1):49-56.

36. Chalasani N, Clark WS, Martin LG, Kamean J, Khan MA, Patel NH, Boyer TD. Determinants of mortality in patients with advanced cirrhosis after transjugular intrahepatic portosystemic shunting. Gastroenterology. 2000; 118(1):138-44.

37. Liu Z, Que S, Xu J, Peng T. Alanine aminotransferase-old biomarker and new concept: a review. Int J Med Sci. 2014;11(9):925-35.

38. Kim HC, Nam CM, Jee SH, Han KH, DK O, Suh I. Normal serum aminotransferase concentration and risk of mortality from liver diseases: prospective cohort study. BMJ. Clin Res ed. 2004;328(7446):983.

39. Ismail FW, Mumtaz K, Shah HA, Hamid S, Abbas Z, Abid S, Anis K, Ahmad A, Jafri W. Factors predicting in-hospital mortality in patients with cirrhosis hospitalized with gastro-esophageal variceal hemorrhage. Indian journal of gastroenterology : official journal of the Indian Society of Gastroenterology. 2006;25(5):240-3.

40. Malinchoc M, Kamath PS, Gordon FD, Peine CJ, Rank J, ter Borg PC. A model to predict poor survival in patients undergoing transjugular intrahepatic portosystemic shunts. Hepatology (Baltimore, Md). 2000;31(4):864-71.

41. Boland BS, Dong MH, Bettencourt R, Barrett-Connor E, Loomba R. Association of Serum Bilirubin with aging and mortality. Journal of clinical and experimental hepatology. 2014;4(1):1-7.

42. Ong KL, Allison MA, Cheung BMY, BJ W, Barter PJ, Rye KA. The relationship between Total bilirubin levels and Total mortality in older adults: the United States National Health and nutrition examination survey (NHANES) 19992004. Plos One. 2014;9(4)

43. Breimer LH, Wannamethee G, Ebrahim S, Shaper AG. Serum bilirubin and risk of ischemic heart disease in middle-aged British men. Clin Chem. 1995; 41(10):1504-8

44. Okuhara K, Kisaka T, Ozono R, Kurisu S, Inoue I, Soga J, Yano Y, Oshima T, Kihara Y, Yoshizumi M. Change in bilirubin level following acute myocardial infarction is an index for heme oxygenase activation. South Med J. 2010; 103(9):876-81.

45. Barkun A, Bardou M, Marshall JK. Consensus recommendations for managing patients with nonvariceal upper gastrointestinal bleeding. Ann Intern Med. 2003;139(10):843-57.

46. Baradarian R, Ramdhaney S, Chapalamadugu R, Skoczylas L, Wang K, Rivilis S, Remus K, Mayer I, Iswara K, Tenner S. Early intensive resuscitation of patients with upper gastrointestinal bleeding decreases mortality. Am J Gastroenterol. 2004;99(4):619-22.

\section{Submit your next manuscript to BioMed Central and we will help you at every step:}

- We accept pre-submission inquiries

- Our selector tool helps you to find the most relevant journal

- We provide round the clock customer support

- Convenient online submission

- Thorough peer review

- Inclusion in PubMed and all major indexing services

- Maximum visibility for your research

Submit your manuscript at www.biomedcentral.com/submit 